\title{
Nuevos datos sobre el paisaje vegetal de las primeras ocupaciones de Mallorca: el Coval Simó (Escorca, Mallorca)*
}

\author{
New data about the landscape of the first occupation of Mallorca: Coval Simó (Escorca, \\ Mallorca)
}

\author{
Yolanda Carrión Marco ${ }^{\mathrm{a}}$, Guillem Pérez-Jordàb, Jaume Coll Conesa ${ }^{\mathrm{c}}$ y Damià Ramis ${ }^{\mathrm{d}}$
}

\section{RESUMEN}

El Coval Simó constituye una de las evidencias más antiguas de poblamiento en la isla de Mallorca y en el archipiélago balear. Tiene, además, la particularidad de ser un hábitat en zona de montaña, de modo que los grupos humanos que se asentaron allí debieron de adaptar su sistema agropecuario y de explotación del entorno a este medio. Los restos vegetales (carbones y semillas) recuperados en los niveles de ocupación del yacimiento permiten aproximarse a estas cuestiones, ya que son resultado de las distintas actividades desarrolladas en esta cavidad: combustible para las actividades domésticas, alimento para el ganado, etc. Los resultados de este estudio muestran que entre el III y II milenio cal BC se implantó un sistema agropecuario basado en la ganadería y en el cultivo de cereales, que realizaba una explotación de las formaciones forestales locales para la obtención de recursos, entre ellos, el combustible leñoso. Las formaciones vegetales explotadas remiten a la existencia de bosques de enebros o sabinas, con presencia de arces y matorrales de leguminosas, entre otros arbustos y matas.

\begin{abstract}
The Coval Simo shelter provides some of the oldest evidence for settlement on the island of Mallorca and the Balearic archipelago. It also has the peculiarity of being a habitat in a mountain area, so that the human groups that settled there had to adapt their agricultural and farming system to this environment. The plant remains (wood charcoal
\end{abstract}

and seeds) recovered in the occupation levels allow us to address these issues, since they are the result of the different activities developed in this cavity: fuel for domestic activities, food for livestock, etc. The results of this study show that between the III and II millennium cal BC, an agricultural system based on livestock and cereal farming was implemented, which exploited local forest formations to obtain resources, among them, firewood. The plants that were exploited show the existence of juniper forests, with the presence of maples and legumes, among other shrubs and bushes.

Palabras clave: Isla de Mallorca; Campaniforme; Primer poblamiento estable; Macrorrestos vegetales; Paisaje de montaña; Sistema agropecuario; Prehistoria Reciente.

Key words: Majorca Island; Bell Beaker; First settlement; Plant macroremains; Mountain landscape; Farming system; Late Prehistory.

\section{INTRODUCCIÓN}

Los ecosistemas insulares conforman paisajes aislados, frágiles $\mathrm{y}$, al mismo tiempo, con un enorme potencial para medir el impacto de la actividad humana sobre ellos. Son especialmente interesantes las secuencias correspondientes a las primeras ocupaciones en un territorio, ya que permiten valorar la progresiva transformación de un paisaje prístino en uno humani-

\footnotetext{
* Este trabajo está subvencionado por el Consell Insular de Mallorca (campaña de excavación) y por el Proyecto "La acción humana como agente transformador del paisaje: análisis de macrorrestos vegetales en las cuatro grandes islas de Baleares" (SESILLES) (HAR2017-83656P, Ministerio de Economía, Industria y Competitividad).

a Prehistoria del Mediterráneo Occidental -PREMEDOC- GIUV2015-213. Universitat de València. Dept. de Prehistòria, Arqueologia i Història Antiga. Av. Blasco Ibañez 28. 46010 València. España. Correo e.: yolanda.carrion@uv.es https://orcid.org/0000-0003-4064-249X

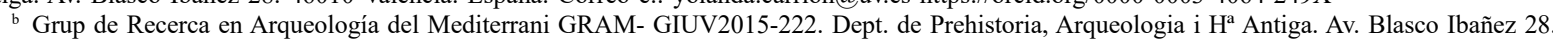
46010 València. España. Correo e.: guillem.perez@uv.es https://orcid.org/0000-0003-1459-0219

c Museo Nacional de Cerámica “González Martí”. C/ Poeta Querol 2. 46002 València. España. Correo e.: jaume.coll@cultura.gob.es https://orcid.org/0000-0002-5849-8345

d Investigador independiente C/ Josep Maria Quadrado, 32,07760 Ciutadella de Menorca, Correo e.: damiaramis@gmail.com https://orcid.org/0000-0002-8940-1956

Recibido 9-IV-2019; aceptado 10-VII-2019.
}

Copyright: (C) 2020 CSIC. Este es un artículo de acceso abierto distribuido bajo los términos de la licencia de uso y distribución "Creative Commons Reconocimiento 4.0 Internacional” (CC BY 4.0) 
zado (Badal et al. 2017). Las Islas Baleares son un archipiélago que fue colonizado por los humanos de forma tardía, durante la Prehistoria reciente y, aunque comparte algunos elementos culturales con el continente europeo, es evidente el rumbo independiente que adquieren las culturas en este territorio. Desde la primera ocupación de las islas, los humanos han tenido que adaptarse al medio insular. Las particularidades de cada isla, su paisaje y escala, en ocasiones, han supuesto un hándicap para el poblamiento y el desarrollo de los sistemas económicos humanos (Ramis 2014).

Existe cierto consenso en las cronologías del primer poblamiento estable en Mallorca, que se documenta desde finales del III milenio a. n. e. (Ramis et al. 2002; Alcover 2008; Ramis 2010; Coll y Ramis 2014), con evidencias tanto de habitación en cueva, como al aire libre (Waldren 1984; Coll 2000, 2001, 2010; Coll y Ramis 2015, entre otros), que entrarían dentro del marco cultural del Calcolítico/Campaniforme. Este tardío poblamiento de uno de los principales territorios insulares del Mediterráneo, que hace que las Baleares cuenten con una prehistoria de poco más de dos milenios, representa una destacada singularidad en este contexto (e. g. Cherry y Leppard 2018). No obstante, se debate la cronología y naturaleza del primer poblamiento de las Islas Baleares. La colonización humana de una isla en general, y de Mallorca en particular, debe entenderse como un proceso. Algunos autores (Guerrero et al. 2007; Guerrero y Calvo 2008; Servera-Vives et al. 2018) defienden la existencia de una fase de frecuentaciones de más de un milenio de duración anterior a la "ocupación permanente" de las Islas Baleares, y que se remontaría al IV milenio a. n. e. No obstante, resulta un tanto difícil aceptar que unos grupos humanos basados en prácticas agropastorales, aunque fueran de carácter móvil, se limitaran a frecuentar las islas de Mallorca y Menorca desde el continente europeo, situado a casi 200 $\mathrm{km}$, sin establecerse en ellas. Así, la mayoría de autores que se han aproximado durante los últimos años a la cuestión del primer poblamiento de las Baleares no considera que hubiera una fase con entidad propia previa a la ocupación definitiva de las islas (Broodbank 2006, 2013; Alcover 2008; Ramis 2010, 2014; Lull et al. 2013, 2014; Dawson 2014; Bover et al. 2016; Cherry y Leppard 2018, entre otros). Los estudios polínicos se unen a este debate al detectar los primeros signos de impacto antrópico en el paisaje en momentos pre-Calcolíticos (Burjachs et al. 2017; Servera-Vives et al. 2018). Consisten en reducciones puntuales del polen arbóreo y aparición de especies ligadas a la actividad humana. No obstante, los propios autores reconocen que es difícil esclarecer si algunos de estos episodios tienen origen antrópico o climático, ya que el cambio más importante que se detecta, p. ej., en la secuencia de Alcúdia (Mallorca), coincide también con un evento climático de acusada aridez (ca. 2300 cal BC) siendo las evidencias anteriores mucho más tenues (Burjachs et al. 2017: 853).

A esta discusión se añade otra acerca de la adecuación del tipo de restos escogidos para la datación, apostando preferentemente por la fauna doméstica, previamente identificada, y procedente de contextos sedimentarios no alterados, valorando, además, las fechas en conjunto con el resto de la información arqueológica y ecológica (Coll y Ramis 2014). En este sentido, las dataciones sobre polen son también problemáticas, ya que estos restos no se datan de forma directa.

Los estudios paleoambientales resultan especialmente reveladores para la reconstrucción de la dinámica del primer poblamiento, sobre todo los que se basan en restos que permiten una valoración del paisaje a nivel local, en el entorno inmediato a los lugares de actividad humana. Una visión muy generalista puede enmascarar las diferencias que, seguro, existen en el territorio balear. Los macrorrestos vegetales son muy adecuados para desvelar el paisaje explotado por los humanos, ya que son aportes antrópicos, por tanto, culturales, desde el entorno del hábitat. Los primeros lugares de ocupación de un territorio son fundamentales para obtener una visión del paisaje prístino que encontraron los pobladores, como demuestran los espectros de macrorrestos vegetales (carbones y semillas). La acción humana resulta visible tras algunos siglos de ocupación continuada (Badal et al. 2012).

La escasez de datos arqueobotánicos que aún persiste en las Islas Baleares deja enormes lagunas para obtener una secuencia de vegetación de este archipiélago en su conjunto y, sobre todo, en lo que se refiere a las primeras ocupaciones. Los estudios de macrorrestos se localizan principalmente en zonas costeras y se inician a partir de la Edad del Bronce (Piqué y Noguera 2002, 2003; Picornell-Gelabert 2012; Picornell-Gelabert et al. 2013; Picornell-Gelabert y Carrión Marco 2017; Picornell-Gelabert y Servera-Vives 2017; Pérez-Jordà et al. 2018). Otros yacimientos más o menos contemporáneos, como Son Olesa (Waldren 1987), Ca Na Cotxera (Cantarellas 1972) o S'Arenalet de Son Colom (Ramis et al. 2007; Ramis 2010), carecen por el momento de análisis paleobotánicos que permitan trazar un mapa detallado del paisaje de las primeras ocupaciones de la isla. La única excepción, por el momento, son sendos análisis antracológicos en Son Matge y Son Gallard, en la misma Serra de Tramuntana (Picornell-Gelabert et al. 2010). El Coval Simó, además de contribuir a aumentar la escasa información para este periodo, ofrece la oportunidad de valorar la explotación de las zonas de montaña, integrando los resultados en la problemática sobre el primer poblamiento humano de las Baleares y su impacto real en el paisaje de la isla. 


\section{EL YACIMIENTO DEL COVAL SIMÓ}

El Coval Simó $\left(6^{\circ} 28^{\prime} 18^{\prime \prime}\right.$ E, 3947’23” N) es un abrigo localizado a $920 \mathrm{~m}$ de altitud en la sierra de Tramuntana, entre el valle de Son Torrella y el llano de Cúber, que forma parte de un sistema de dos grandes dolinas cársticas excavadas en el macizo rocoso (Alcover et al. 2001) (Fig. 1). El abrigo se abre con orientación NE en una dolina de unos $400 \mathrm{~m}^{2}$. Estas formaciones presentan una sedimentación natural muy horizontal y con un elevado índice de retención de humedad, indicada por los juncos y encinas sobre ellas (Coll 2001). La localización del abrigo es estratégica, ya que tiene acceso al valle y a la zona escarpada inmediata, con diversos recursos explotables. Hay suelos erosionados y afloramientos rocosos predominantes en torno al abrigo, pero la cercanía de pequeños valles, a una distancia de 20 a 40 minutos a pie, ofrece tierras de cultivo en sus alrededores.

Tras una primera incursión en el yacimiento durante la década de los 1960 (Enseñat 1969), los trabajos durante los años 1998 a 2008 (Coll 2010) se centraron en áreas selladas por el desprendimiento de grandes bloques de piedra del techo que las protegieron de alteraciones posteriores.

La excavación bajo los bloques identificó tres horizontes de ocupación (Fig. 2). El Horizonte 3 (H3), un paquete sedimentario de unos $22 \mathrm{~cm}$ de espesor, es el inmediato inferior. El Horizonte 2 (H2), separado del anterior por una capa de piedras más pequeñas, presentaba aproximadamente $16 \mathrm{~cm}$ de espesor. Por debajo se encuentra la primera ocupación humana del refugio, el Horizonte 1 (H1).

El registro arqueológico es uniforme en toda la estratigrafia: cerámica campaniforme (Coll 2015), fragmentos de queseras (Coll 2014) y un abundante conjunto faunístico con claro dominio de restos de cabra y oveja (Ramis 2018). Entre los restos de talla en sílex recuperados había algunos relacionados con las denominadas hoces tabulares. Además se hallaron diversos fragmentos de escorias de aspecto vítreo producidas a partir de la reducción de mineral de cobre. Esta se realizaba, probablemente, en cimas próximas al abrigo que poseían vetas de este mineral (Ramis et al. 2005; Coll 2010). Luego estas escorias habrían sido transportadas al yacimiento junto con el sílex, probablemente debido a sus propiedades como material vítreo.

Los conjuntos cerámicos ofrecían la posibilidad de investigar la seriación del Campaniforme en Mallorca, con pocos contextos bien datados hasta este momento. La información cronológica del yacimiento sitúa su ocupación dentro del período $c a$. 2300-1900 cal BC; con dos dataciones situadas en el intervalo $c a$. 2300-2050 cal BC (Beta54196: $3760 \pm 40$ BP, KIA-15726: $3740 \pm 30$ BP) y otras tres en el intervalo $c a$. 2150-1900 cal BC (KIA-14323: $3670 \pm 30$ BP, Beta-161787: $3650 \pm 40$ BP, KIA-29166: $3625 \pm 25 \mathrm{BP})$, a partir de las cuales, se han fechado los tres horizontes citados (Coll y Ramis 2014: fig. 6).
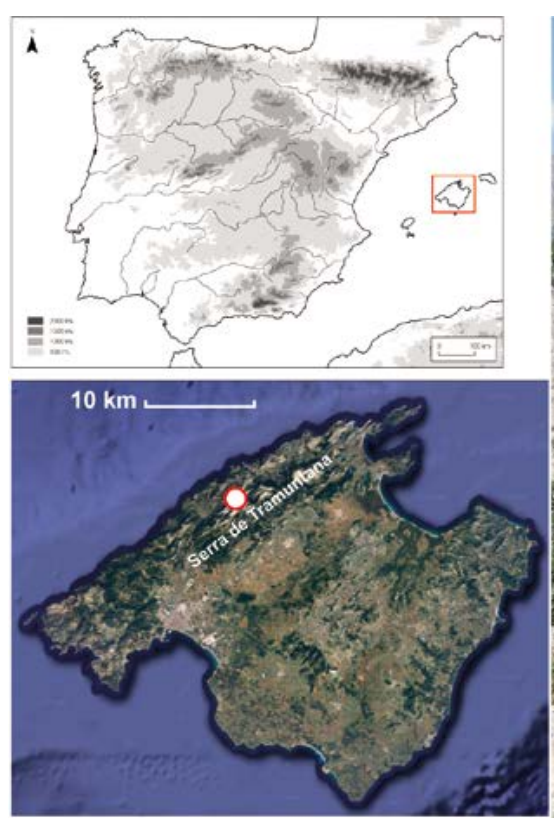

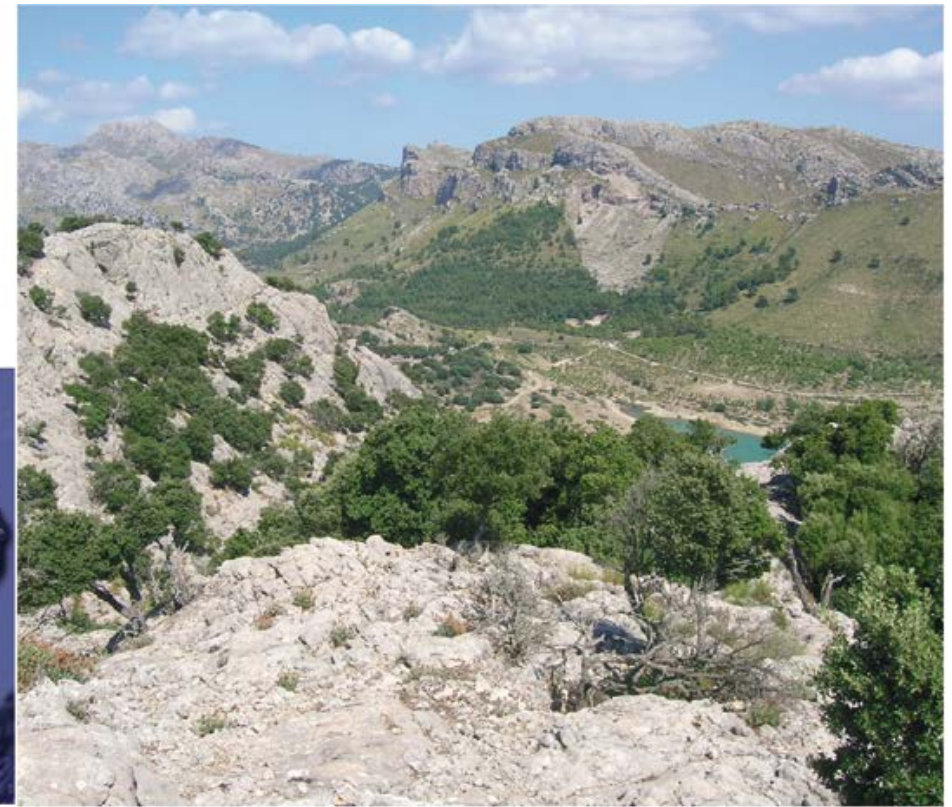

Fig. 1. Localización del Coval Simó (Escorca) en la isla de Mallorca (izquierda). Entorno actual de la Serra de Tramuntana desde la boca del abrigo hacia el noreste (derecha), foto de D. Ramis (en color en la versión electrónica). 


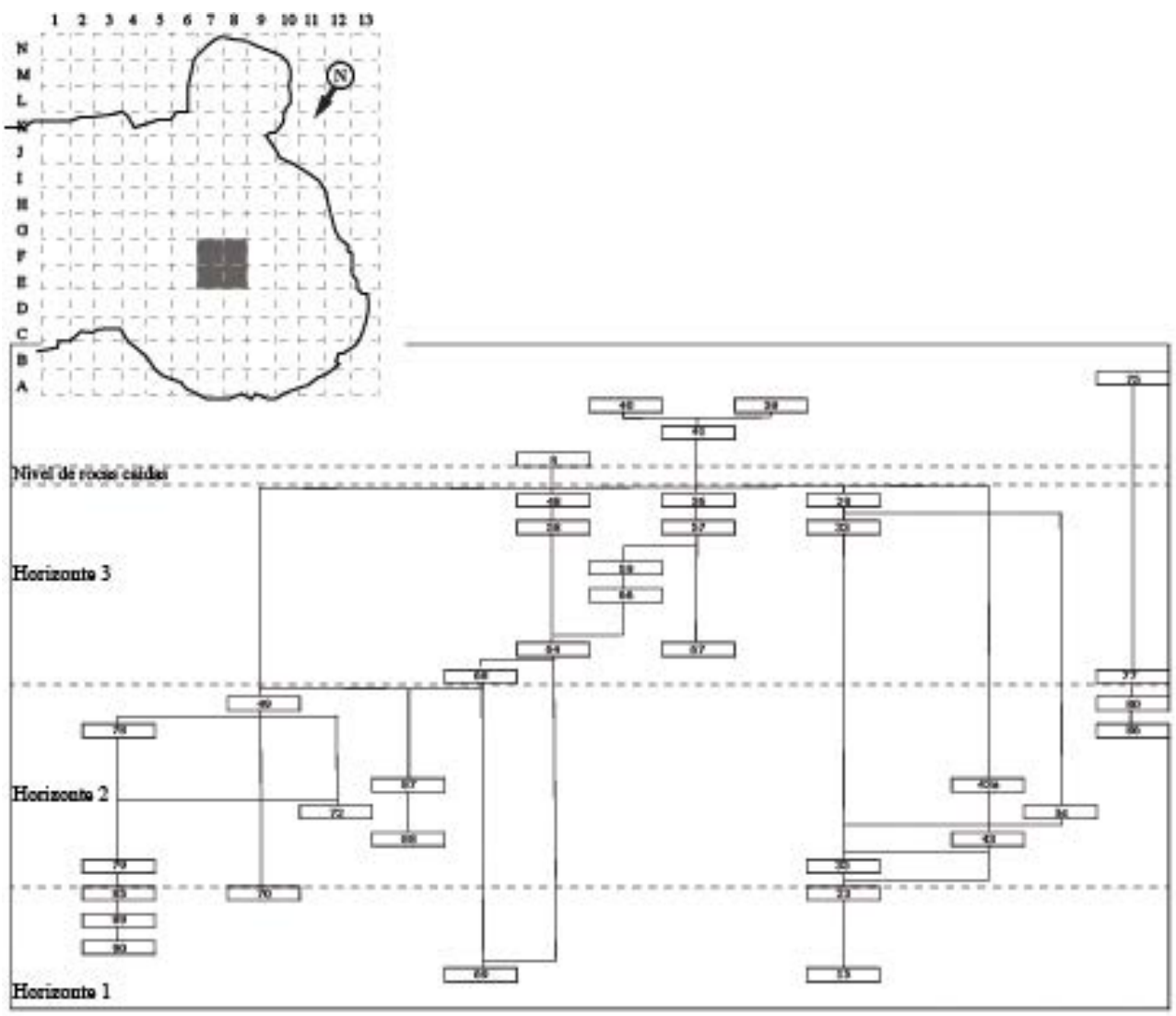

Fig. 2. Interpretación estratigráfica del área central del Coval Simó: cuadros E7-8, F7-8 (sombreados en el plano de la cueva).

\section{MATERIAL Y MÉTODO}

En este artículo presentamos los resultados del análisis de carbones y carporrestos recuperados en el Coval Simó durante las campañas 2001-2003. Las muestras han sido procesadas mediante el cribado en seco o con una máquina de flotación. En el interior de la cuba se colocó una malla de $1 \mathrm{~mm}$, donde se recuperaron los materiales más densos (sílex, microfauna, y algunos carbones y semillas); una malla de $0,25 \mathrm{~mm}$ recogía los restos que flotaban. Posteriormente, los diversos tipos de materiales botánicos de las muestras han sido seleccionados en el laboratorio con la ayuda de una lupa, trabajando entre 3 y 20 aumentos.

El análisis antracológico se basa en la identificación botánica del carbón que permite saber de qué especies vegetales procede el carbón, se ha realizado en el Laboratorio de Arqueología Milagro Gil-Mascarell de la Universitat de València. El carbón se ha observado a través de un microscopio óptico de luz reflejada de campo claro-oscuro, con objetivos que van desde 50 a 1000 aumentos, modelo Leica DM6000 M, y comparado con la colección actual de referencia y/o la bibliografía especializada en anatomía vegetal (Greguss 1955, 1959; Jacquiot 1955; Jacquiot et al. 1973; Schweingruber 1990). Cuando se ha precisado de mayor magnificación o profundidad de campo o de fotografías se ha recurrido a un microscopio electrónico de barrido (MEB) Hitachi S-4100, en el Servei Central de Suport a la Investigació Experimental - SCSIE.

Por su parte, la Paleocarpología se encarga del estudio de las semillas y de los frutos recuperados en contextos arqueológicos. La conservación de estos materiales puede producirse de diversas formas, siendo la más habitual en nuestro caso la carbonización, proceso que en general es accidental. Otra forma de conservación que se ha detectado en las Islas Baleares en el interior de cavidades que presentan unas condiciones ambientales muy estables, es la desecación (Lull et al. 1999). Los estudios paleocarpológicos aportan algunos datos de tipo paleoambiental, pero proporcionan información básicamente paleoeconómica. Por este motivo su desarrollo es la base para conocer las características de la agricultura y de la recolección de semillas y frutos practicada por los habitantes de esta cavidad. 
En el Coval Simó, 37 muestras han proporcionado restos arqueobotánicos: 8 de $\mathrm{H} 1,16$ de $\mathrm{H} 2$ y 13 de H3. Durante las campañas de excavación de 2001 y 2002, se cribó todo el sedimento en seco. En la campaña de 2003 se flotó de forma sistemática una muestra de 101 de cada uno de los cuadros y niveles excavados.
La flotación ha proporcionado un conjunto de semillas y frutos muy escaso. Los carbones son abundantes tanto en las muestras cribadas como en las flotadas, sin que se hayan detectado diferencias en los taxones presentes debidas al sistema de muestreo empleado.

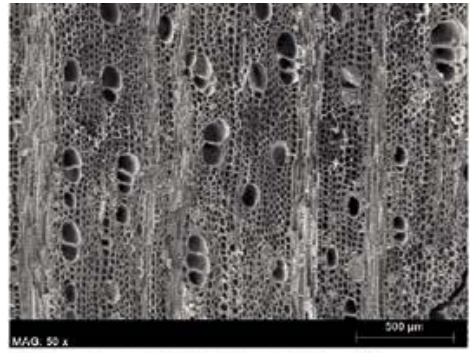

Acer sp., corte transversal x 50

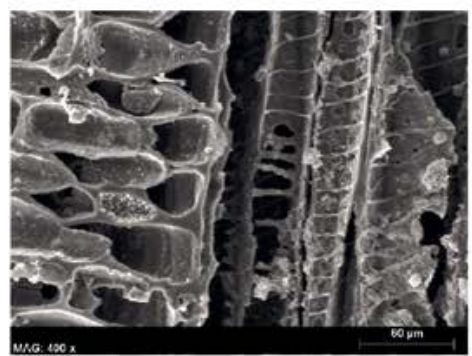

Arbutus unedo, corte radial $\times 400$

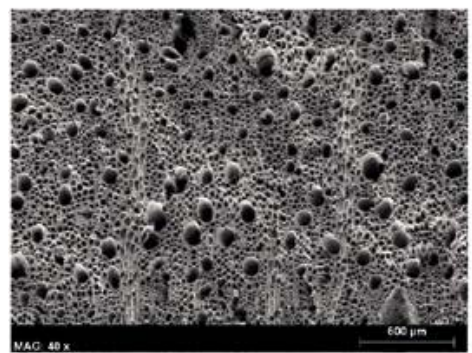

Erica sp., corte transversal $\mathrm{x} 40$

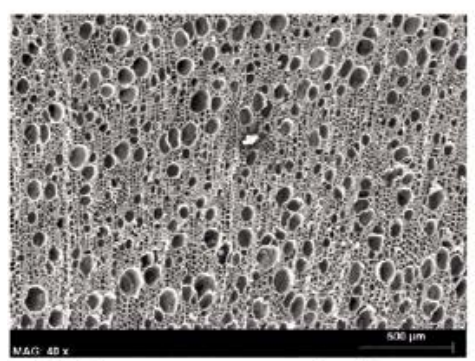

Labiada, corte transversal $\mathrm{x} 40$

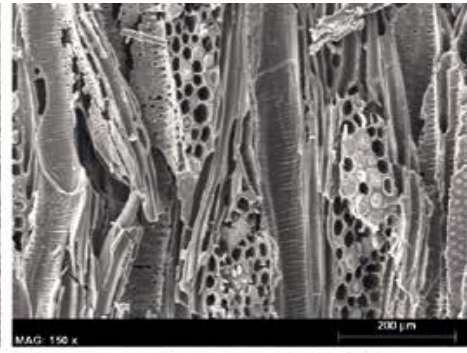

Acer sp., corte tangencial x 150

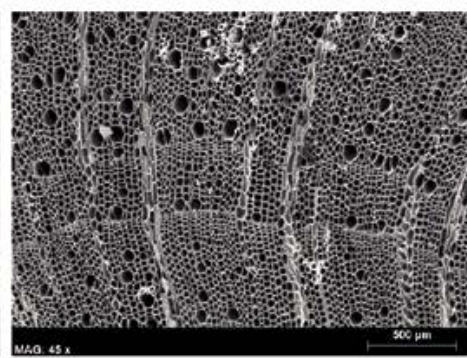

Ephedra sp., corte transversal x45

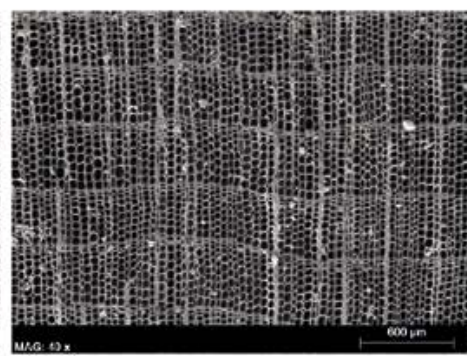

Juniperus $\mathrm{sp}$., corte transversal $\mathrm{x} 40$

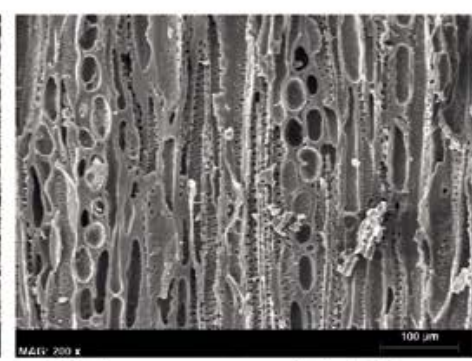

Labiada, corte tangencial x200

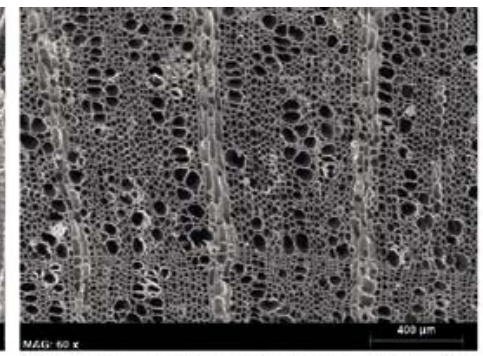

Arbutus unedo, corte transversal x60

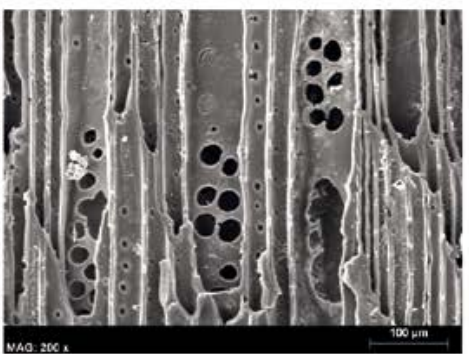

Ephedra sp., corte radial x200

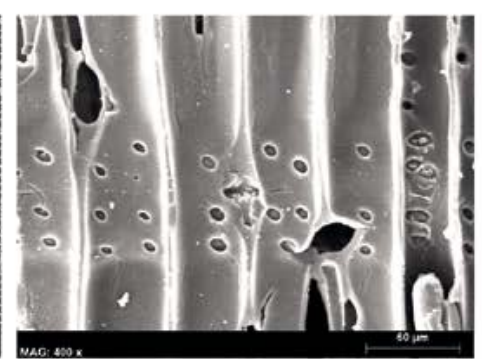

Juniperus sp., corte radial x400

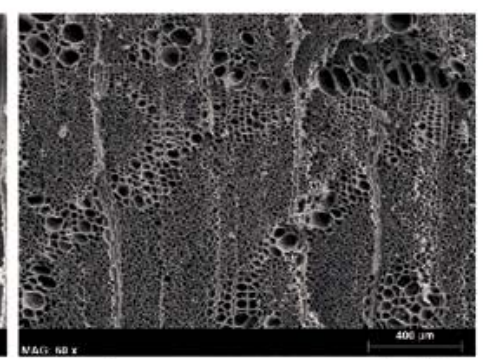

Leguminosa, corte transversal x60

Fig. 3. Fotografías en microscopio electrónico de algunos de los taxones identificados en el carbón del Coval Simó (Escorca, Mallorca).

Trab. Prehist., 77, N. ${ }^{\circ}$ 1, enero-junio 2020, pp. 148-162, ISSN: 0082-5638

https://doi.org/10.3989/tp.2020.12251 


\section{RESULTADOS}

\section{La flora leñosa identificada en los carbones}

Se han analizado un total de 2108 fragmentos de carbón y se han identificado los siguientes taxones leñosos: Acer sp. (arce), Arbutus unedo (madroño), Buxus sp. (boj), Clematis sp. (clemátide), Conífera, Ephedra sp. (belcho, efedra), Erica sp. (brezo), Hedera helix (hiedra), Juniperus sp. (enebro y/o sabina), Labiatae (labiada), Leguminosae (leguminosas, fabáceas), Maloideae (rosácea), Monocotiledónea, Olea europaea (acebuche, olivo), Prunus sp. (del género del cerezo), Quercus perennifolio (carrasca, coscoja), Rhamnus-Phillyrea (aladierno-labiérnago), Rosmarinus officinalis (romero) y Taxus baccata (tejo) (Tab. 1, Figs. 3 y 4). Están representados taxones de especies arbóreas, de matorral y algunas trepadoras. Todos ellos corresponden a la vegetación potencial de las Islas Baleares: un bosque termomediterráneo seco o subhúmedo inferior, tipo encinar (Rivas-Martínez 1987).

Juniperus sp., Acer sp., Leguminosae y RhamnusPhillyrea son los taxones mejor representados y más ubicuos. Aparecen casi sistemáticamente en casi todos los conjuntos analizados (Fig. 5-A), por lo que deberían ser las especies más abundantes y/o accesibles en el entorno del yacimiento para la obtención de leña. Siguen, en porcentaje mucho menor y con presencia más esporádica en las diferentes UUEE, las labiadas, el romero, el acebuche, el tejo, Ephedra y Quercus perennifolio. El resto de taxones no llega al $1 \%$ del total de la madera utilizada. Ello nos lleva a pensar en su explotación más ocasional, que podría también traducir su menor entidad en el paisaje inmediato.

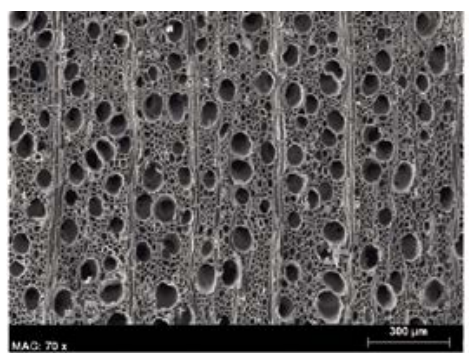

Maloidea, corte transversal x 70

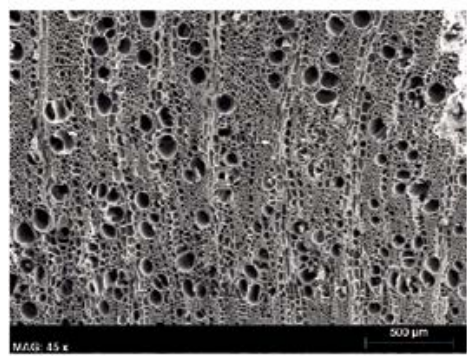

Prumus sp., corte transversal $\mathrm{x} 45$

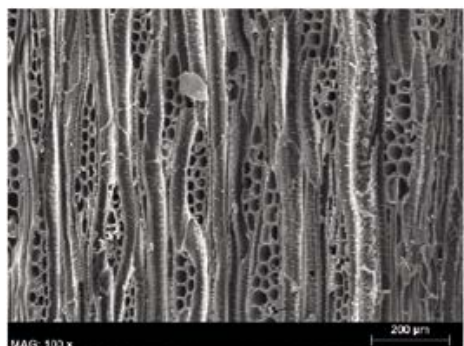

Rhamnus-Phillyrea, corte tangencial $\mathrm{x} 100$

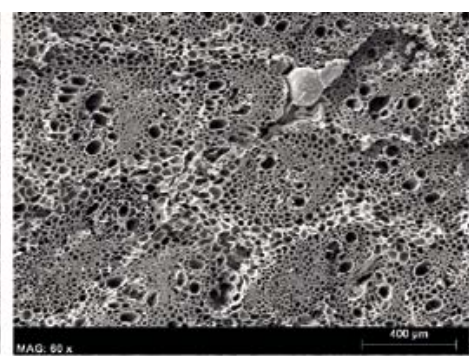

Monocotiledónea, corte transversal x60

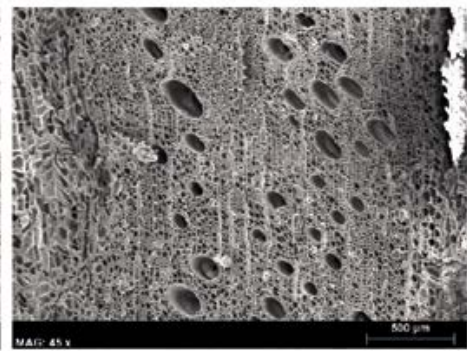

Quercus perennifolio, corte transversal x45

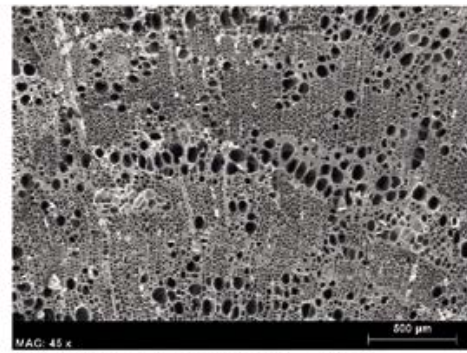

Rosmarimus officinalis, corte transversal $x 45$

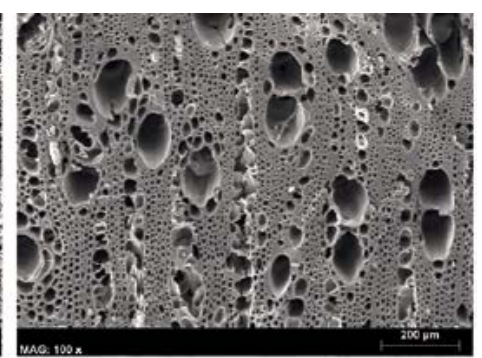

Olea europaea, corte transversal x100

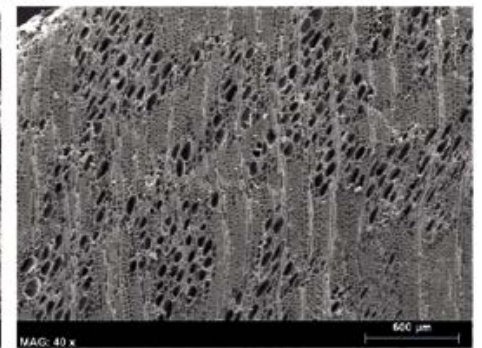

Rhamnus-Phillyrea, corte transversal $\mathrm{x} 40$

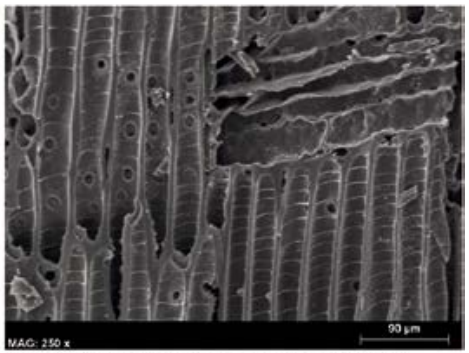

Taxus baccata, corte radial $\times 250$

Fig. 4. Fotografías en microscopio electrónico de algunos de los taxones identificados en el carbón del Coval Simó (Escorca, Mallorca). 
La presencia de taxones en cada muestra analizada no es uniforme. En algún caso esto podría deberse a un desigual número de fragmentos de carbón, aunque no parece existir una relación directa entre el número de carbones analizado y la riqueza taxonómica de las mismas (Fig. 5-B). P. ej., en la Unidad 85 se han analizado 134 fragmentos de carbón y se han identificado 5 taxones, mientras que en la Unidad 68, cuadro E6, con la mitad de fragmentos se han identificado 8 ta- xones (Tab. 1). En la Unidad 77, cuadro F4, se recuperaron sólo 44 fragmentos de carbón, pero se han identificado 8 taxones, entre ellos, la única representación de Clematis en el conjunto de carbón, así como la mayor parte de los fragmentos de Prunus.

El diagrama antracológico (Fig. 6) ofrece una imagen diacrónica de la flora leñosa identificada en los tres horizontes sedimentarios del abrigo. Aparentemente, no hay cambios significativos a lo largo de la se-

A

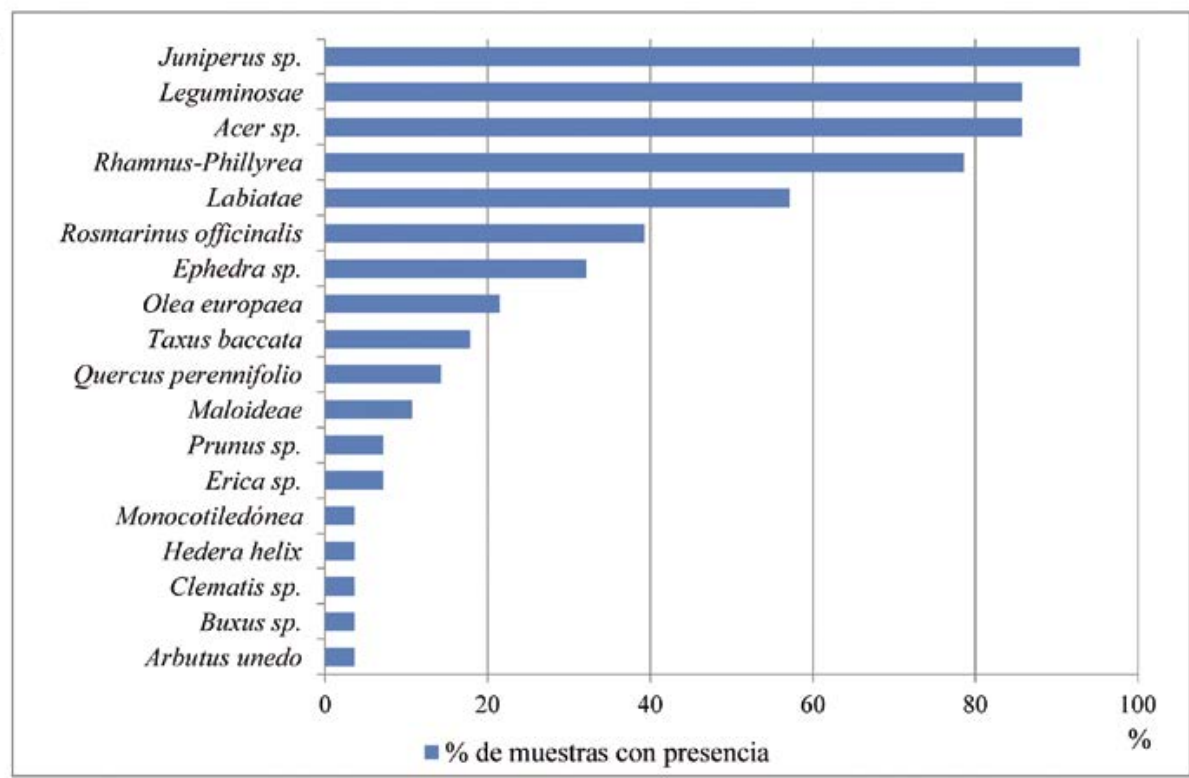

B

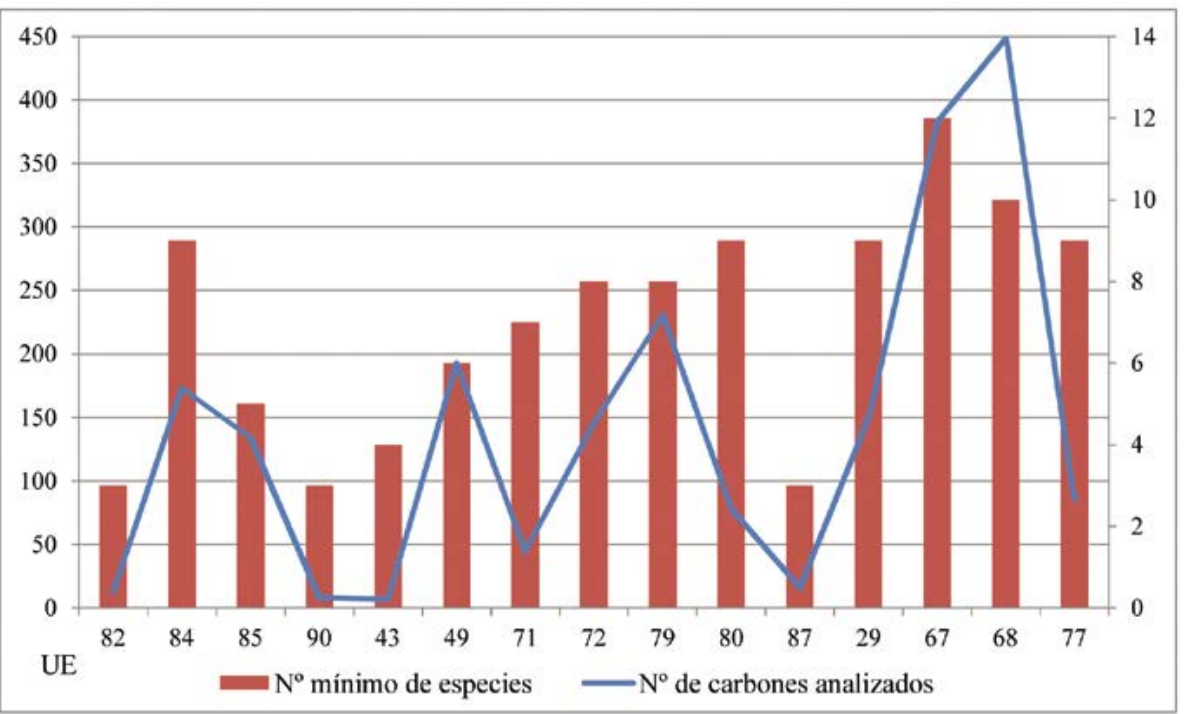

Fig. 5. Coval Simó (Escorca, Mallorca): A. Ubicuidad de los taxones identificados; B. Relación del número de carbones analizados con el número mínimo de especies identificadas (en color en la versión electrónica). 


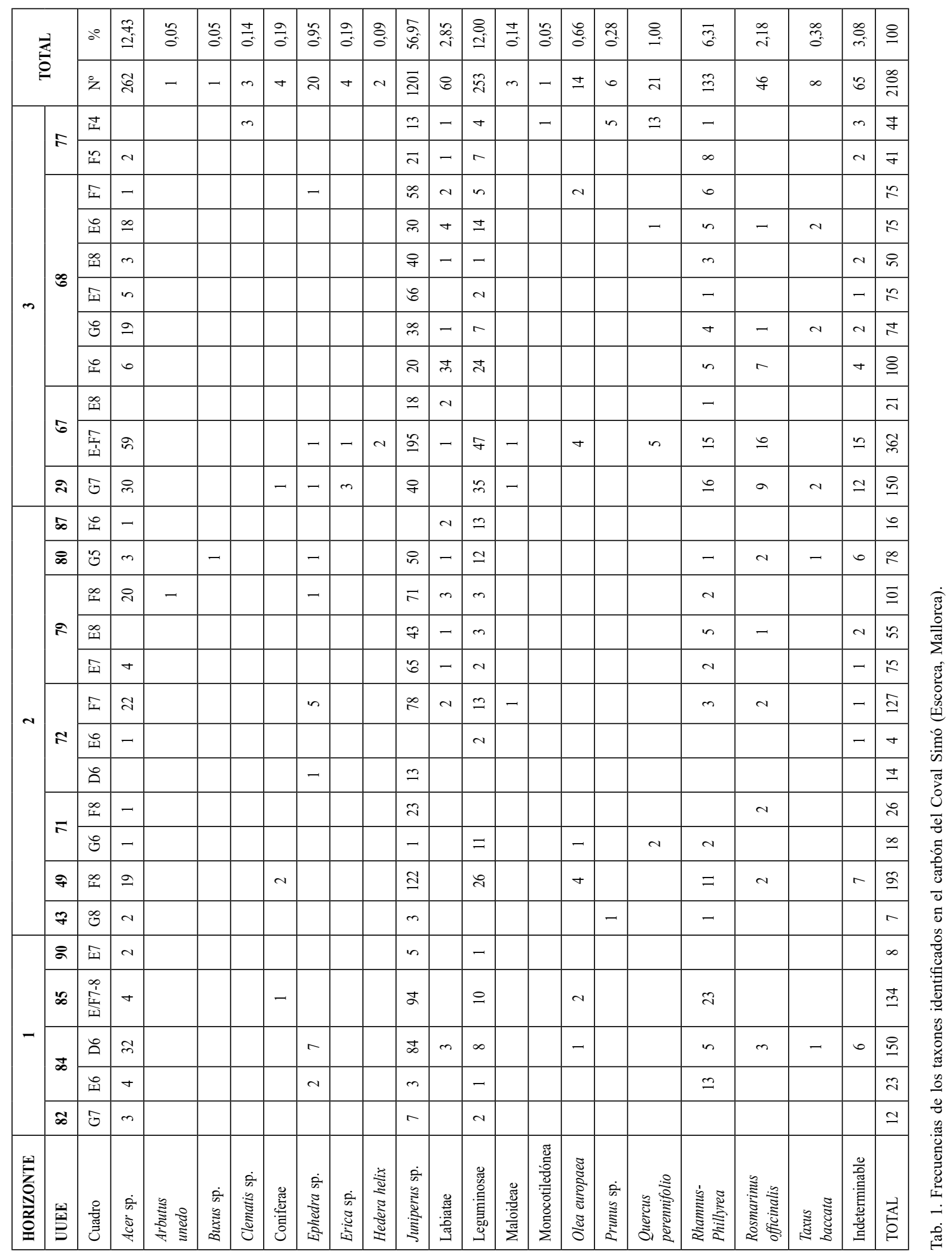

Trab. Prehist., 77, N. ${ }^{\circ}$ 1, enero-junio 2020, pp. 148-162, ISSN: 0082-5638 https://doi.org/10.3989/tp.2020.12251 
cuencia, salvo una ligera tendencia, de base a techo, a la diversificación de las especies de matorral, con un aumento de las leguminosas y las labiadas, así como del grupo de "otros taxones" (Varia), cuya presencia sistemáticamente es muy ocasional (y por debajo del $1 \%)$. Su aparente enriquecimiento taxonómico en el último espectro (Horizonte 3) podría ser resultado de una mayor diversificación de los matorrales en el paisaje. Tampoco podemos descartar que refleje la mayor representatividad del conjunto de carbón de este horizonte. Es cierto (véase más arriba) que no parece haber una relación entre el número de carbones y de taxones en cada muestra. Sin embargo, en el total del Horizonte 3 sí estamos ante un conjunto más representativo no sólo por el número total de carbones analizado, sino también por el número de contextos o muestras, que ofrecerían una imagen más completa del conjunto de especies leñosas utilizado (Chabal 1997). En todo caso, la estabilidad general del diagrama es coherente con el desarrollo de las mismas actividades en el abrigo durante la secuencia y con la frecuentación y estabilidad de las mismas formaciones vegetales durante toda su ocupación.

\section{Resultados carpológicos}

De las 37 muestras flotadas, sólo 6 han aportado algún resto de semilla o fruto. El total de 8 restos conservados da una densidad de materiales muy baja: apenas supera 1 resto por cada 10 litros de sedimento. Es decir, el yacimiento conserva muy escasas semillas y frutos. La única planta cultivada es un cereal, la cebada (Hordeum vulgare), aunque el mal estado de conservación de la única cariópside impide definir si corresponde a las formas desnudas o vestidas. Los ta-
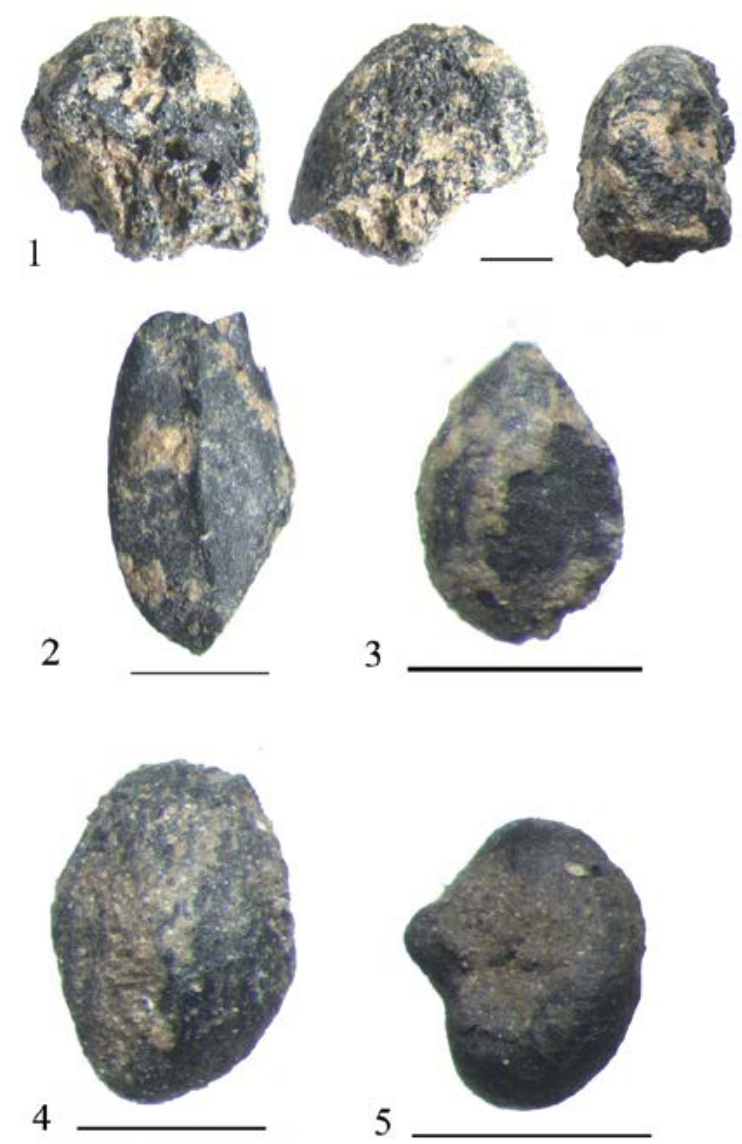

Fig. 7. Restos carpológicos del Coval Simó (Escorca, Mallorca). 1. Hordeum vulgare; 2. Phalaris sp.; 3. Cyperacea; 4. Indeterminada; 5. Melilotus/Trifolium (escala $1 \mathrm{~mm}$ ) (en color en la versión electrónica).

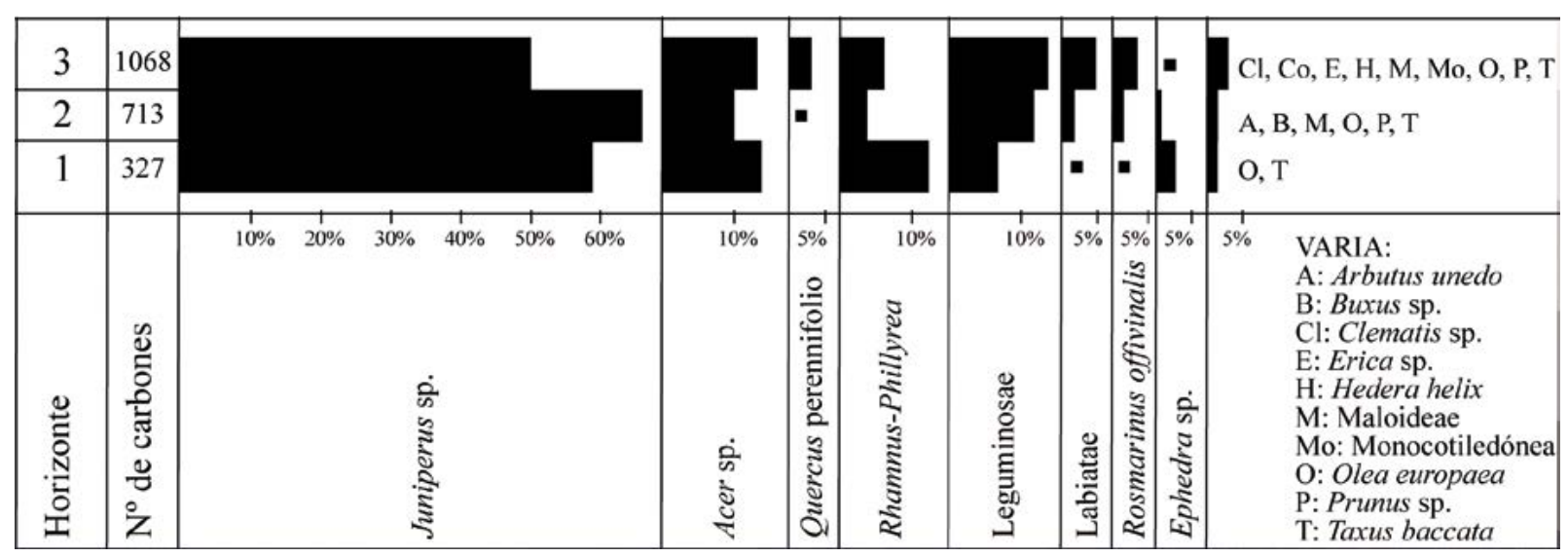

Fig. 6. Diagrama antracológico del Coval Simó (Escorca, Mallorca).

Trab. Prehist., 77, N. ${ }^{\circ}$ 1, enero-junio 2020, pp. 148-162, ISSN: 0082-5638

https://doi.org/10.3989/tp.2020.12251 


\begin{tabular}{|c|c|c|c|c|c|c|c|}
\hline \multirow{3}{*}{$\begin{array}{l}\text { Horizonte } \\
\text { UE } \\
\text { Cuadro }\end{array}$} & \multicolumn{3}{|c|}{2} & \multicolumn{3}{|c|}{3} & \multirow{3}{*}{ Total } \\
\hline & \multirow{2}{*}{\begin{tabular}{|c|}
43 \\
G-8
\end{tabular}} & \multicolumn{2}{|c|}{79} & \multicolumn{2}{|c|}{68} & \multirow{2}{*}{$\begin{array}{c}77 \\
\text { F-4 }\end{array}$} & \\
\hline & & E-7 & F-8 & E-6 & E-7 & & \\
\hline Volumen 1. & 10 & 10 & 10 & 10 & 10 & 10 & 60 \\
\hline $\begin{array}{l}\text { Hordeum } \\
\text { vulgare frag. }\end{array}$ & & & & & 1 & & 1 \\
\hline Cyperacea & & & & 1 & & & 1 \\
\hline $\begin{array}{l}\text { Melilotus/ } \\
\text { Trifolium }\end{array}$ & & & & 1 & & & 1 \\
\hline Phalaris sp. & & & 1 & & & & 1 \\
\hline cf Prunus sp. & 1 & & & & & & 1 \\
\hline Indeterminada & & & & & & 1 & 1 \\
\hline $\begin{array}{l}\text { Indeterminada } \\
\text { frag. }\end{array}$ & & 1 & & & & & 1 \\
\hline $\mathrm{N}^{\mathrm{o}}$ de restos & 1 & 1 & 1 & 2 & 1 & 1 & 8 \\
\hline $\begin{array}{l}\text { Densidad x } 10 \\
\text { 1. }\end{array}$ & 1 & 1 & 1 & 2 & 1 & 1 & 1,3 \\
\hline $\mathrm{N}^{0}$ de taxones & 1 & 1 & 1 & 2 & 1 & 1 & 5 \\
\hline
\end{tabular}

Tab. 2. Materiales carpológicos recuperados en las muestras (Escorca, Mallorca) (1.= litros; UE = Unidad estratigráfica; frag. = fragmento).

xones de las plantas silvestres son: Cyperacea, Melilotus/Trifolium, Phalaris sp. y cf. Prunus. (Tab. 2, Fig. 7). Un resto y un fragmento no han podido ser determinados. Las plantas en su mayoría son herbáceas, en principio, sin utilidad alimenticia. Taxones como Cyperaceae (ciperácea), Melilotus/Trifolium (tréboles) o Phalaris sp. engloban especies que pueden crecer como malas hierbas en los campos de cultivo generalmente de cereales, así como plantas desarrolladas en el entorno de las zonas de hábitat, ayudadas por la acumulación de desechos que la actividad humana genera. Son taxones habituales en el registro arqueobotánico mediterráneo.

Otro taxón que pudo tener un uso alimenticio es cf. Prunus sp., detectado igualmente entre los restos de madera carbonizada. Esta asociación entre carbones y frutos plantea dos posibilidades: una recolección intencionada de los frutos o una presencia accidental al haber recogido como combustible para los hogares, ramas de este árbol que conservaran adheridos alguno de sus frutos. Hay que valorar en todo caso que el fruto aparece en el Horizonte 2 y los restos de madera en el 3.

Como en el estudio de la madera carbonizada, tampoco se aprecian diferencias entre los Horizontes 2 y 3 , que han aportado los restos de semillas y frutos. La pobreza del registro limita sus posibilidades de lectura.

\section{DISCUSIÓN}

\section{El paisaje del Coval Simó entre el III y II milenio cal BC}

Los estudios sobre vegetación de las Islas Baleares se han basado tradicionalmente en secuencias de polen que, además, han permitido remontarse a tiempos anteriores a la presencia humana en las islas. Estas secuencias han marcado el establecimiento de los paisajes litorales de carácter termomediterráneo (con formaciones de acebuche y lentisco), datados, al menos, desde el Holoceno medio en la mayor parte de las secuencias (Burjachs et al. 1994, 2017; Pérez-Obiol et al. 1996; Pantaleón-Cano et al. 2000; Servera-Vives et al. 2018). Los análisis polínicos comienzan a detectar entre 3500-2100 cal BC los primeros indicios de impacto de la actividad humana en el paisaje con el retroceso de algunas especies autóctonas, como el boj, coincidiendo con la aparición del polen de cereal y de otras plantas ruderales (Burjachs et al. 1994; 2017; Yll et al. 1994, 1997, 1999; Pantaleón-Cano et al. 2000; Servera-Vives et al. 2018). Será en cronologías ya del Calcolítico cuando se produzca la clara expansión de especies esclerófilas de matorral (acebuche y lentisco) y la desaparición de otros taxones mesófilos (Burjachs et al. 2016; Servera-Vives et al. 2018).

La imagen que aporta el polen es matizada a nivel local por los crecientes estudios de macrorrestos vegetales, en su mayoría centrados en zonas litorales, que indican la presencia de formaciones esclerófilas termófilas con pinos, acebuche, lentisco, madroño, jaras, brezos, mirto y algunas sabinas, entre otros (Piqué y Noguera 2002; Picornell-Gelabert y Carrión 2017; Picornell-Gelabert y Servera-Vives 2017). Los datos del Coval Simó complementan la imagen local de la vegetación de montaña en la isla, para la que existen datos escasos y aislados (Picornell-Gelabert et al. 2010), pero coherentes con los aquí obtenidos.

El conjunto de taxones identificados en los macrorrestos vegetales del Coval Simó ofrece una información ecológica bastante precisa. Los requerimientos ambientales (temperatura, humedad, suelo) de cada uno muestran una distribución de rango bastante amplio, desde los matorrales sublitorales termomediterráneos hasta zonas de montaña. El yacimiento se sitúa en una zona que permite el acceso a ambos ecosistemas, siendo más relevante la vegetación perteneciente al segundo de ellos, en coherencia con la localización de la cavidad, en la umbría de una de las estribaciones de la Serra de Tramuntana (Coll y Ramis 2014).

Actualmente Juniperus es uno de los géneros más presentes en el entorno del Coval Simó, ya que estas especies se adaptan a zonas escarpadas con escaso desarrollo edáfico. Se confirma su presencia importante 
desde el Campaniforme, como también se documenta de forma dominante en otros yacimientos más o menos contemporáneos, como Son Gallard y Son Matge (Picornell-Gelabert et al. 2010). A partir de las características anatómicas de su madera, no se pueden identificar las especies, que se desarrollan desde el nivel del mar hasta los $1500 \mathrm{~m}$ de altitud en las montañas ibéricas. En la isla de Mallorca, los enebros y sabinas constituyen una formación de transición a las zonas montañosas, sobre todo en macizos calcáreos entre 700-1000 m de altitud, donde cobra especial importancia la serie Buxo-Juniperetum phoeniceae, es decir, del boj con sabina negral (Pérez-Obiol et al. 1996).

En el carbón del Coval Simó, la presencia de arce revela también la existencia de zonas umbrosas, con vegetación arbórea y suelos bien desarrollados. Para algunos autores es indicadora de formaciones de transición (Pérez-Obiol et al. 1996). Concretamente, la serie Aceri-Buxetum balearicae juega un notable papel en la región montañosa septentrional de Mallorca a más de $1000 \mathrm{~m}$. Los estudios polínicos datan el descenso del boj hacia el 2500 cal BC (Burjachs et al. 2017). La escasa importancia del boj en el carbón del Coval Simó puede corresponder a una fase en la que esta especie ya ha perdido importancia en el paisaje de la isla.

Este momento también coincide con una reducción drástica de los enebros en las secuencias polínicas litorales, sustituidos por matorrales de acebuche y lentisco (Burjachs et al. 1994, 2017). A partir de entonces, parece que la presencia de Juniperus pasa a ser indicadora exclusivamente de los citados ambientes de transición a las zonas de montaña. Ello corrobora la argumentación inicial que planteamos, de que se explotan mayoritariamente ambientes montañosos, apareciendo sólo un eco de formaciones litorales (con Olea europaea, Ephedra, Phillyrea, Erica, etc.).

En Coval Simó, la vegetación arbustiva, representada por las leguminosas, es escasa. En zonas más bajas, los análisis paleobotánicos muestran cierta importancia de los matorrales de Erica como componente fundamental de las maquias litorales (Yll et al. 1994). Posiblemente, este hecho marque una diferencia importante entre la vegetación de las zonas litorales y las montañosas, donde se da probablemente un mayor desarrollo de las leguminosas.

Destacamos también, en los macrorrestos del Coval Simó, la total ausencia de pinos, elementos típicamente mediterráneos ampliamente extendidos en otras zonas de la isla y en cronologías posteriores (PicornellGelabert y Carrión 2017). Esta ausencia se constata en algunas secuencias polínicas (Burjachs et al. 1994; Pérez-Obiol et al. 1996; Yll et al. 1999) incluso en momentos posteriores al comienzo de la acción antrópica sobre el medio, cuando este género manifiesta su máxima expansión en territorios peninsulares costeros
(Badal y Bernabeu 1990; Bernabeu et al. 1994; Badal 2009; Carrión y Grau 2015). Esta dinámica podría explicarse por la falta de pinos en la zona de captación de leña del Coval Simó y por su rápida expansión en cronologías posteriores. Esta cuestión necesita mayor investigación a la luz de futuras secuencias botánicas.

En las secuencias antracológicas disponibles para las Islas Baleares y, más concretamente, para la isla de Mallorca, sí se observan ciertas coincidencias con los taxones identificados en el Coval Simó: es frecuente la presencia de Juniperus, Rhamnus-Phillyrea, Erica sp., Leguminosae, Olea europaea, Rosmarinus officinalis o Pistacia lentiscus, entre otros (Piqué y Noguera 2002; Picornell-Gelabert et al. 2010; Picornell-Gelabert y Carrión 2017; Picornell-Gelabert y Servera-Vives 2017).

En síntesis, la vegetación leñosa documentada en el Coval Simó es coherente con la localización geográfica de la cavidad, descartando un acceso frecuente a zonas más bajas para el abastecimiento de leña. El que sólo se documente un "eco" de formaciones termófilas en el registro demuestra una explotación del entorno local. Este elenco de especies explotadas, podría ser también coherente con las actividades concretas desarrolladas en la cavidad, como se comentará en el siguiente apartado.

\section{Usos de las plantas silvestres en el yacimiento}

La presencia de leña y otros órganos vegetativos en un yacimiento ofrece una imagen de las especies presentes en el entorno siempre sometida a cierto sesgo cultural. No obstante, la recurrencia de la ocupación $\mathrm{y}$, por tanto, del aporte de plantas acaba constituyendo una muestra representativa de la vegetación del área de captación del sitio (Chabal 1997).

En el Coval Simó, el conjunto de materiales arqueológicos apunta a prácticas de carácter doméstico y de uso de la cavidad como lugar de estabulación de ganado, dos actividades que están en directa relación con el uso de plantas. Todos los restos de madera proceden de carbones dispersos por los niveles arqueológicos. Presumiblemente son restos de combustible, sin descartar el uso como combustible de plantas con un interés económico, artesanal, medicinal, etc. Los fuegos domésticos no suelen utilizar combustible especializado, sino lo que está más a mano en el entorno del hábitat. Como el abanico de especies disponibles en el entorno bastaría para procurarse leña, aun asumiendo el componente cultural existente en su recolección, esta da una imagen fiable y ecológicamente coherente de las formaciones vegetales presentes en el entorno del yacimiento, como se ha mostrado en el apartado anterior. 
En Coval Simó no aparecen niveles estratigráficos de tipo fumier, indicativos de actividad ganadera, pero el alto porcentaje de premolares de leche de cabra y oveja (una cuarta parte del total de molariformes) sugiere el uso del abrigo como lugar de estabulación. Este porcentaje se asemeja al obtenido en la muestra estudiada del abrigo de Son Matge, que prácticamente dobla el de los yacimientos situados en el llano de la isla. Además, las edades de sacrificio de los animales domésticos del Coval Simó hacen pensar en una ocupación intermitente del abrigo, posiblemente de carácter estacional ${ }^{1}$.

Es conocido el uso especializado de ciertas especies para la alimentación tradicional del ganado. P. ej., las hojas y el ramón de arce juegan un papel destacado en las sociedades ganaderas tradicionales, por su alto contenido en materia grasa, en agua y por el alto porcentaje de hoja por rama (Pardini y Nori 2011; Émile et al. 2016). El aporte de ramón explicaría la presencia de madera carbonizada, que podría quemarse ocasionalmente para limpieza del lugar, o reaprovecharse como combustible, una vez consumidas sus hojas (Badal 1999; Bergadà et al. 2005). Ello podría explicar la abundancia de este taxón tan escaso en otros registros paleobotánicos de la época. La misma hipótesis se puede aplicar al hallazgo de Juniperus, aparentemente una planta menos apetecible. Sin embargo la etnografía demuestra que, en general, algunas especies de sabina son apreciadas como buen forraje (se distinguen entre sabinas amargas y dulces). Su rama, denominada barda o ramonizo, es valorada como tal para las ovejas. Ello concuerda con la cabaña identificada en Coval Simó, en la que predominan los ovicápridos. El carbón de sabina está también documentado en los niveles de estabulación de Son Matge, junto a otras plantas de tradición forrajera, como el boj (PicornellGelabert et al. 2010).

El aporte como forraje de ciertas especies al Coval Simó podría hacer que estas estuvieran sobrerrepresentadas en el registro antracológico. Sin embargo no pensamos que eso altere sustancialmente la imagen que hemos propuesto para la reconstrucción de la vegetación. De haber utilizado plantas de un radio superior al local, no se explicaría la exclusión de plantas muy palatables, como Olea europaea (Badal 1999), muy abundantes en cotas más bajas de la isla (Burjachs et al. 2017; Picornell-Gelabert y Carrión 2017), y que, curiosamente, también faltan en los niveles de corral de Son Matge (Picornell-Gelabert et al. 2010).

D. Ramis. Estudio faunistico de las fases iniciales de la Prehistoria de Mallorca. Tesis doctoral inédita Universidad Nacional de Educación a Distancia, Madrid 2006: 416-417.

\section{Semillas y frutos como indicadores de la actividad humana y animal}

El registro carpológico de Coval Simó, que fue usada fundamentalmente como lugar de estabulación (Coll y Ramis 2014: 445) es, como se ha indicado, bastante pobre. Es cierto que, en las cavidades utilizadas con esta funcionalidad, se dan registros carpológicos muy dispares. Los de la Cueva del Mirador (Atapuerca, Burgos) (Rodríguez et al. 2016) o los de Ifri Oudadane (Marruecos) (Morales et al. 2013) aportan conjuntos muy ricos en semillas y frutos. Otros del País Valenciano, como la Cova de les Cendres (Buxó i Capdevila 1997), les Coves de Santa Maira o la Cova d'En Pardo (Pérez-Jordà 2013) y l'Abric de Falguera (Pérez-Jordà 2006) presentan un registro más bien pobre.

Los registros de la Cova de les Cendres y de la Cova de l'Or (Pérez-Jordà 2013) son ejemplos de los cambios que se observan en el material arqueobotánico dependiendo del uso de estos espacios. En los niveles antiguos de ambos, cuando las cavidades sirven como lugares de hábitat, hay un registro rico, mientras que, a partir del V milenio cal BC, cuando pasan a ser utilizadas como lugares de estabulación, la densidad de restos carpológicos es mucho más pobre. Coval Simó tiene un comportamiento muy similar a las cavidades documentadas en el País Valenciano (PérezJordà 2006, 2013).

En Coval Simó la presencia de plantas cultivadas se limita a una cariópside de cebada. El resto son taxones sin uso alimenticio salvo, quizás, un fruto de Prunus sp. Estos pueden haber llegado a la cavidad al ser utilizados como combustible, como malas hierbas de los cereales, o por estar vinculados a los ovicápridos. Cyperacea, Phalaris y Melilotus/Trifolium son semillas de pequeño tamaño, de plantas habitualmente consumidas por el ganado, por lo que pueden proceder de los excrementos depositados durante su estabulación en la cueva.

El hecho más relevante del registro carpológico de esta cavidad es la constatación del cultivo de cereales ya en el tránsito entre el III y el II milenio cal BC que, por el momento, establece la más antigua práctica de la agricultura en el archipiélago balear (PérezJordà et al. 2018). Solo Coval Simó y el conjunto de materiales recuperados en la Cova des Riuets (Formentera) (López Garí et al. 2013) aportan información sobre estas primeras fases de ocupación de las Islas Baleares. En el primero no es posible confirmar si la cebada corresponde a las variedades vestida o desnuda. En el yacimiento de Formentera domina la cebada desnuda pero aparecen también los trigos desnudos.

Con estos datos se puede pensar que el sistema agrícola de los agricultores que se establecen en estas 
islas es muy similar al detectado en este momento en la franja oriental de la Península Ibérica (Buxó i Capdevila 1997; Rovira i Buendía 2007; Pérez-Jordà 2013; Antolín 2016). Allí los cultivos de las variedades desnudas de trigos y cebadas dominan con claridad la actividad agrícola de estos grupos. Con todo es muy escaso lo que conocemos de las prácticas agrícolas de estas comunidades. Por el momento sólo es posible sugerir su mayor similitud a las del área de Cataluña y del Sur de Francia (Pérez-Jordà et al. 2018), coincidiendo con otros elementos del registro arqueológico (Lull et al. 2004; Alcover 2008; Ramis 2010). Se pone así de manifiesto la necesidad de un muestreo más intenso en niveles campaniformes, para conocer mejor los cultivos desarrollados por estos primeros grupos de agricultores.

\section{CONCLUSIONES}

El análisis del carbón y las semillas recuperadas en Coval Simó da cuenta de las actividades humanas en relación con la explotación de los recursos vegetales en sentido amplio. Se pone en evidencia la frecuentación del territorio de su entorno para la obtención de estos recursos, destinados a la alimentación humana y del ganado, y como combustible doméstico.

El registro antracológico representa perfectamente la vegetación de las vertientes de las sierras donde se localiza Coval Simó: la de transición de las zonas sublitorales a la media/alta montaña. Están ausentes o poco representadas las especies características de las zonas bajas termomediterráneas y dominan las formaciones de enebros y sabinas, arces y leguminosas sobre todo, con un estrato de matorral bastante rico, aunque poco representado. Esta escasez puede deberse a una orientación hacia maderas de calibre medio-grande y/o a determinadas especies, en relación con las actividades que se desarrollaron en la cueva, esto es, un leñateo orientado a la obtención de combustible para los hogares domésticos, y de plantas forrajeras.

Quizás estemos ante la explotación de las formaciones vegetales más inmediatas al abrigo, esto es, de las vertientes de la propia cavidad, más desprotegidas (expuestas a la insolación, vientos, etc.) o con menor desarrollo edáfico. Allí las sabinas y leguminosas juegan un papel fundamental, mientras que la vegetación arbórea o con mayores necesidades edáficas se desarrollaría en enclaves más aptos (umbrías, pequeñas vaguadas, etc.).

Una parte de los materiales vegetales procede de los llanos cultivables próximos (Coma de Son Torrella, a $873 \mathrm{~m}$ ) o Cúber (a $750 \mathrm{~m}$ ). Allí es presumible que se cultivaran los cereales que los ocupantes de Coval Simó subirían para asegurar su alimentación. Alguno de los frutos, como la rosácea, también podría estar reflejando una explotación de los frutos comestibles que crecen en el entorno de la cueva. Se trata, por lo tanto, de un registro típico donde el uso de los recursos cultivados se combina con la explotación de aquellos taxones silvestres que puedan complementar la dieta.

Esta secuencia ha permitido constatar el cultivo de cereales ya en el tránsito entre el III y el II milenio cal $\mathrm{BC}$, repitiendo un patrón de explotación del territorio (agrícola y ganadero), que vemos en otras regiones y que tarda entre 500 y 1000 años en dejar su huella en el paisaje (Badal et al. 2017). La secuencia del Coval Simó no tiene suficiente duración para poder apreciar la antropización del paisaje, pero sí permite constatar una explotación intensa, agrícola y de leñateo para fuegos domésticos y/o metalúrgicos del entorno más inmediato al yacimiento durante la primera ocupación del lugar.

\section{BIBLIOGRAFÍA}

Alcover, J. 2008: "The first Mallorcans: prehistoric colonization in the Western Mediterranean". Journal of World Prehistory 21: 19-84. https://doi.org/10.1007/s10963-008-9010-2

Alcover, J. A.; Ramis, D.; Coll, J. y Trias, M. 2001: "Bases per al coneixement del contacte entre els primers colonitzadors humans i la naturalesa de les Balears". Endins 24: 5-57.

Antolín, F. 2016: Local, intensive and diverse? Early farmers and plant economy in the North-East of the Iberian Peninsula (5500-2300 cal $B C)$. Advances in Archaeobotany 2, Barkhuis. Groningen.

Badal, E. 1999: "El potencial pecuario de la vegetación mediterránea: las cuevas redil". En J. Bernabeu Aubán y T. Orozco Köhler (eds.): Actes del II Congrés del Neolític a la Península Ibèrica (Valencia 1999). Saguntum. Papeles del Laboratorio de Arqueología de Valencia, Extra 2. Valencia: 69-75.

Badal, E. 2009: "Estudio antracológico de la secuencia holocena de la cova de les Cendres". En J. Bernabeu y L1. Molina Balaguer (eds.): La Cova de Les Cendres (Moraira-Teulada, Alicante). Museo Arqueológico de Alicante - MARQ, Serie Mayor 6. Alicante: 125-134.

Badal, E. y Bernabeu, J. 1990: "Imagen de la vegetación y utilización económica del bosque en los asentamientos neolíticos de Jovades y Niuet (Alicante)". Archivo de Prehistoria Levantina 20: 143-166.

Badal, E.; Carrión Marco, Y.; Chabal, L.; Figueiral, I. y Thiébault, S. 2017: "Neolithic Human Societies and woodlands in the north-western Mediterranean region. Wood and charcoal analysis". En O. García Puchol y D. C. Salazar García (eds.): Times of Neolithic Transition along Western Mediterranean. Fundamental Issues in Archaeology series, Springer. Cham, Switzerland: 135-169.

Badal, E.; Martí, B. y Pérez-Ripoll, M. 2012: "From agricultural to pastoral use: changes in neolithic landscape at Cova de l'Or (Alicante, Spain)". En E. Badal, Y. Carrión, M. Macías y M. Ntinou (eds.): Wood and charcoal. Evidence for human and natural history. Saguntum. Papeles del Laboratorio de Arqueología de Valencia, Extra 13. Valencia: $75-84$.

Bergadà, M.; Guerreo, V. M.; Ensenyat, J. 2005: "Primeras evidencias de estabulación en el yacimiento de Son Matge (Serra de Tramuntana, Mallorca) a través del registro sedimentario". Mayurqa 30: 153-180.

Bernabeu, J.; Pascual Benito, J. L1.; García Puchol, O.; Fumanal García, M. a P. y Badal, E. 1994: "Niuet (l'Alqueria d'Asnar). Poblado del III milenio a. C.”. Recerques del Museu d'Alcoi 3: 9-74. 
Bover, P.; Valenzuela, A.; Torres, E.; Cooper, A.; Pons, J. y Alcover, A. 2016: "Closing the gap: new data on the last documented Myotragus and the first human evidence on Mallorca (Balearic Islands, Western Mediterranean Sea)". The Holocene 26 (11): 1887-1891. https://doi.org/10.1177/0959683616645945

Broodbank, C. 2006: "The origins and early development of Mediterranean maritime activity". Journal of Mediterranean Archaeology 19 (2): 199-230. https://doi.org/10.1558//jmea.2006.v19i2.199

Broodbank, C. 2013: The making of the Middle Sea: a history of the Mediterranean from the beginning to the emergence of the Classical World. Thames and Hudson. London.

Burjachs, F.; Pérez-Obiol, R.; Picornell-Gelabert, L.; Revelles, J.; Servera-Vives, G.; Expósito, I. e Yll, E. I. 2016: "Changements environnementaux et histoire de la colonisation humaine des Îles Baléares (Méditerranée Occidentale): conséquences sur l'évolution de la végétation". En M. Ghilardi (ed.): Géoarchéologie des îles de Méditerranée. CNRS. Alpha: 259-272.

Burjachs, F.; Pérez-Obiol, R.; Picornell-Gelabert, L1.; Revelles, J.; Servera-Vives, G.; Expósito, I. e Y11, E. I. 2017: "Overview of environmental changes and human colonization in the Balearic Islands (Western Mediterranean) and their impacts on vegetation composition during the Holocene". Journal of Archaeological Science: Reports 12: 845-859. https://doi.org/10.1016/j.jasrep.2016.09.018

Burjachs, F.; Pérez-Obiol, R.; Roure, J. M. y Julià, R. 1994: “Dinámica de la vegetación durante el Holoceno en la Isla de Mallorca". En I. Mateu, M. Dupré, J. Güemes y M. E. Burgaz (eds.): Trabajos de Palinología básica y aplicada. X Simposio de Palinología (APLE). Universitat de València. Valencia: 199-210.

Buxó i Capdevila, R. 1997: Arqueología de las plantas: la explotación económica de las semillas y los frutos en el marco mediterráneo de la Península Ibérica. Crítica/Arqueología. Barcelona.

Cantarellas, C. 1972: “Excavaciones en 'Ca Na Cotxera' (Muro, Mallorca)". En Noticiario Arqueológico Hispánico - Prehistoria 1. Servicio de Publicaciones del Ministerio de Educación y Ciencia. Madrid: 179-226.

Carrión Marco, Y. y Grau Almero, E. 2015: "Madera y leña en la Lloma

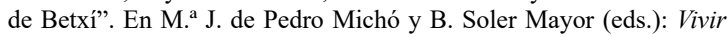
junto al Turia hace 4000 años. La Lloma de Betxí. Museo de Prehistoria de Valencia, Diputación Provincial de Valencia. Valencia: 62-67.

Chabal, L. 1997: Forêts et sociétés en Languedoc (Néolithique final, Antiquité tardive), L'anthracologie, méthode et paléoécologie. Documents d'Archéologie Française 63, Maison des Sciences de l'Homme. Paris.

Cherry, J. F. y Leppard, T. H. 2018: "The balearic paradox: why were the islands colonized so late?" Pyrenae 49: 49-70. http://doi.org/ 10.1344/Pyrenae2018.vol49num1.2

Coll Conesa, J. 2000: "Excavación arqueológica del Coval Simó. Estado actual y perspectivas de futuro". En V. M. Guerrero y S. Gornés (eds.): Colonización humana en ambientes insulares. Interacción con el medio y adaptación cultural. Universitat de les Illes Balears. Palma: 371-400.

Coll Conesa, J. 2001: "Primeres datacions absolutes del jaciment de Coval Simó". Endins 24: 161-8.

Coll Conesa, J. 2010: "Memòria preliminar de les d'excavacions arqueològiques del coval Simó (Escorca). Resultats generals (2008)". En F. Tugores, A. Lozano y C. Andreu (eds.): Memòria del Patrimoni Cultural. MPC08. Departament de Cultura i Patrimoni, Consell de Mallorca. Palma.

Coll Conesa, J. 2014: "Los vasos perforados o encellas del período campaniforme en el Coval Simó (Escorca, Mallorca, Illes Balears)". En C. Ferrando y B. Costa (eds.): In Amicitia. Miscel-lània d'Estudis en Homenatge a Jordi H. Fernández. Treballs del Museu Arqueològic d'Eivissa i Formentera 72, Conselleria d'Educació, Cultura i Universitats, Govern de les Illes Balears. Eivissa: 149-162.

Coll Conesa, J. 2015: “Cerámicas impresas e incisas del período del vaso campaniforme halladas en el Coval Simó (Escorca, Mallorca, Illes Balears)". En C. Andreu, C. Ferrando y O. Pons (eds.) L'Entreteixit del Temps. Miscel-lània d'Estudis en Homenatge a Lluís Plantalamor Massanet. Conselleria de Participació, Transparència i Cultura, Govern de les Illes Balears. Palma: 111-123.
Coll Conesa, J. y Ramis, D. 2014: "Absolute chronology for the Beaker Culture site of Coval Simó (Mallorca, Balearic Islands)". Radiocarbon 55(2): 439-450. https://doi.org/10.1017/s0033822200049493

Coll Conesa, J. y Ramis, D. 2015: "Un assentament a l'aire lliure del III mil-lenni a.C. a Son Danús Vell (Santanyí)". En I Jornades d'Estudis Locals de Santanyi (Santanyi 2014): 109-124. Santanyí.

Dawson, H. 2014: Mediterranean voyages. The Archaeology of island colonisation and abandonment. Left Coast Press. Walnut Creek.

Émile, J. C.; Delagarde, R.; Barre, P. y Novak, S. 2016: "Nutritive value and degradability of leaves from temperate woody resources for feeding ruminants in summer". En $3^{\text {rd }}$ European Agroforestry Conference. INRA (Montpellier, France 2016): 409-412. Montpellier.

Enseñat, B. 1969: "Aportación al conocimiento de los primitivos pobladores de Mallorca”. En X Congreso Nacional de Arqueología (Mahón 1967): 67-74. Zaragoza

Greguss, P. 1955: Identification of living Gymnosperms on the basis of Xylotomy. Akadémiai Kiado. Budapest.

Greguss, P. 1959: Holzanatomie der Europaïschen Laubhölzer und Straücher. Akadémiai Kiado. Budapest.

Guerrero Ayuso, V. y Calvo Trias, M. 2008: "Resolviendo incertidumbres. Nuevos datos sobre las primeras ocupaciones humanas de las Baleares”. En M. S. Hernández Pérez, J. A. Soler Díaz y J. A. López Padilla (eds.): IV Congreso del Neolitico Peninsular (Alicante 2006): 331-339. Alicante.

Guerrero Ayuso, V.; Calvo Trias, M.; García Rosselló, J. y Gornés Hachero, S. 2007: Prehistoria de las Islas Baleares: registro arqueológico y evolución social antes de la Edad del Hierro. British Archaeological Reports International Series 1690. Archaeopress. Oxford.

Jacquiot, C. 1955: Atlas d'anatomie des bois des conifères. Centre technique du bois. Paris.

Jacquiot, C.; Trenard, Y. y Dirol, D. 1973: Atlas d'anatomie des bois des angiospermes (Essences feuillues). Centre technique du bois. Paris.

López Garí, J. M.; Pérez-Jordà, G.; Marlasca Martín, R.; Farrera Fernández, V. y Enrich Hoja, J. 2013: "La primera agricultura Pitiusa y Balear: las evidencias de la Cova des Riuets". Saguntum: Papeles del Laboratorio de Arqueología de Valencia 45: 65-77.

Lull, V.; Micó, R.; Rihuete, C. y Risch, R. 1999: Ideología y sociedad en la Prehistoria de Menorca: La Cova des Càrritx y La Cova des Mussol. Consell Insular de Menorca. Menorca.

Lull, V.; Micó, R.; Rihuete, C. y Risch, R. 2004: "Los cambios sociales en las Islas Baleares a lo largo del II milenio". Cypsela 15: 123-148.

Lull, V.; Micó, R.; Rihuete, C. y Risch, R. 2013: “The Bronze Age in the Balearic Islands". En H. Fokkens y A. Harding (ed.): The Oxford Handbook of the European Bronze Age. Oxford University Press. Oxford: 617-631. https://doi.org/10.1093/oxfordhb/9780199572861.013.0034

Lull, V.; Micó, R.; Rihuete, C. y Risch, R. 2014. "Las Islas Baleares: desde la colonización humana estable hasta la conquista romana". En M. Almagro Gorbea (ed.): Protohistoria de la Península Ibérica: del Neolítico a la Romanización. Universidad de Burgos; Fundación Atapuerca. Burgos: 147-159.

Morales, J.; Pérez-Jordà, G.; Peña-Chocarro, L.; Zapata, L.; Ruíz-Alonso, M.; López-Sáez, J. A. y Linstädter, J. 2013: "The origins of agriculture in North-West Africa: macro-botanical remains from Epipalaeolithic and Early Neolithic levels of Ifri Oudadane (Morocco)". Journal of Archaeological Science 40 (6): 2659-2669. https://doi.org/10.1016/j.jas.2013.01.026

Pantaleón-Cano, J.; Roure, J. M. y Pérez-Obiol, R. 2000: "Evaluación de los impactos antrópicos y los cambios climáticos en el paisaje vegetal de las Islas Baleares durante los últimos 8000 años”. En V. M. Guerrero Ayuso y S. Gornés (eds.): Colonización humana en ambientes insulares: interacción con el medio y adaptación cultural. Universitat de les Illes Balears. Palma: 73-98.

Pardini, A. y Nori, M. 2011: "Agro-silvo-pastoral systems in Italy: integration and diversification". Pastoralism 1: 26. https://doi.org/10.1186/2041-7136-1-26.

Pérez-Jordà, G. 2006: "Estudi de les llavors i dels fruits". En O. García Puchol y Ll. Molina Balaguer (eds): El abric de la Falguera (Alcoi, Alacant): 8.000 años de ocupación humana en la cabecera del rio 
de Alcoi 2. Diputación de Alicante, Excmo. Ayuntamiento de Alcoy y Caja de Ahorros del Mediterráneo. Alcoi: 111-119.

Pérez-Jordà, G. 2013: La agricultura en el País Valenciano entre el VI y el I milenio a.C. Tesis Doctoral, Universitat de València. València. http://hdl.handle.net/10550/31152

Pérez-Jordà, G.; Peña-Chocarro, L.; Picornell-Gelabert, Ll. y Carrión Marco, Y. 2018: "Agriculture between the $3^{\text {rd }}$ and $1^{\text {st }}$ millennium BC in the Balearic Islands: the archaeobotanical data". Vegetation History and Archaeobotany 27: 253-265. https://doi.org/10.1007/s00334-017-0618-y

Pérez-Obiol, R.; Y11, E. I.; Pantaleón-Cano, J. y Roure, M. 1996: "Historia de Buxus y Corylus en las Islas Baleares durante el Holoceno". En P. Ramil-Rego, C. Fernández Rodríguez y M. Rodríguez Guitián (eds.): Biogeografia Pleistocena-Holocena de la Península Ibérica. Xunta de Galicia. Santiago: 87-97.

Picornell-Gelabert, L1. 2012: Paisaje vegetal y comunidades prehistóricas y protohistóricas en Mallorca y Menorca (Illes Balears): una aproximación desde la antracología. Tesis Doctoral, Universitat de Barcelona. Barcelona. http://hdl.handle.net/10803/85874

Picornell-Gelabert, L1. y Carrión Marco, Y. 2017: "Landscape and firewood procurement at the prehistoric site of Ses Païsses (Mallorca Island, Western Mediterranean)". Quaternary International 458: 5674. https://doi.org/10.1016/j.quaint.2017.03.018

Picornell-Gelabert, L1.; Guerrero, V. M. y Calvo Trías, M. 2010: “Anàlisis antracològiques a Son Matge i Son Gallard (Valldemossa, Mallorca). Algunes hipòtesis sobre la dinàmica de la vegetació i l'explotació forestal durant el Calcolític a Mallorca". Mayurqa 33: 315-332.

Picornell-Gelabert, L1. y Servera-Vives, G. 2017: "Landscape practices and everyday life in domestic spaces in Bronze Age Mallorca (Balearic Islands): perspectives for and archaeology of fuel and firewood". Quaternary International 431 (A): 73-89. https://doi.org/10.1016/j.quaint.2015.12.058

Picornell-Gelabert, Ll.; Servera-Vives, G.; Riera, S. y Allué, E. 2013: "Archaeobotanical research in the prehistoric Balearic Islands: landscape changes and cultural plant uses". En F. Damblon (ed.): Proceedings of the Fourth International Meeting of Anthracology (Brussels 2008) Royal Belgian Institute of Natural Sciences. British Archaeological Reports International Series. Archaeopress. Oxford: 183-194.

Piqué, R. y Noguera, M. 2002: "Landscape and management of forest resources in the Balearic Islands during the II-I millennium BC". En W. Waldren y J. Ensenyat (eds.): World Islands in prehistory. International insular investigations. British Archaeological Reports International Series. Archaeopress. Oxford: 292-300.

Piqué, R. y Noguera, M. 2003: "La gestión de los recursos forestales durante la prehistoria de las Islas Baleares: el yacimiento del Puig Morter de Son Ferragut". En P. Castro, T. Escoriza y M. E. Sanahuja (eds.): Mujeres y hombres en espacios domésticos. Trabajo y vida social en Mallorca (c. 700-500 cal ANE). El Edificio Alfa del Puig Morter de Son Ferragut (Sineu, Mallorca). British Archaeological Reports International Series, Archaeopress. Oxford: 322-332.

Ramis, D. 2010: "From colonisation to habitation: early cultural adaptations in the Balearic Bronze Age". En P. Van Dommelen y A. B. Knapp (eds.): Material connections in the Ancient Mediterranean: mobility, materiality and Mediterranean identities. Routledge. London: 64-84.

Ramis, D. 2014: "Early island exploitations: productive and subsistence strategies on the Prehistoric Balearic Islands". En A. B. Knapp y P. Van Dommelen (eds.): The Cambridge Prehistory of the Bronze and
Iron Age Mediterranean. Cambridge University Press. Nueva York: 40-56.

Ramis, D. 2018: “Animal Exploitation in the Early Prehistory of the Balearic Islands". Journal of Island and Coastal Archaeology 13: 269-282.

Ramis, D.; Alcover, J. A.; Coll, J. y Trias, M. 2002: “The chronology of the first settlement of the Balearic Islands". Journal of Mediterranean Archaeology 15: 3-24. https://doi.org/10.1558/jmea.v15i1.3

Ramis, D.; Hauptmann, A. y Coll, J. 2005: "Réduction du minerai de cuivre dans la Préhistoire de Majorque". En P. Ambert y J. Vaquer (eds.): Colloque International, La première métallurgie en France et dans les pays limitrophes (Carcassonne 2002). Societé Préhistorique de France. París: 217-224.

Ramis, D.; Plantalamor, Ll.; Carreras, J.; Trías, M. y Santandreu, G. 2007: 'S'Arenalet de Son Colom (Artà) i l'origen de l'arquitectura ciclòpia a les Balears". Bolleti de la Societat Arqueològica Lul-liana: Revista d'estudis històrics 63: 333-348.

Rivas-Martínez, S. 1987: Memoria del mapa de series de vegetación de España 1:400.000. ICONA. Madrid.

Rodríguez, A.; Allué E. y Buxó R. 2016: “Agriculture and livestock economy among prehistoric herders based on plant macro-remains from El Mirador (Atapuerca, Burgos)". Quaternary International 414: 272-284. https://doi.org/10.1016/j.quaint.2016.01.045

Rovira i Buendía, N. 2007: Agricultura y gestión de los recursos vegetales en el sureste de la Península Ibérica durante la prehistoria reciente. Tesis Doctoral, Universitat Pompeu Fabra. Barcelona. http://hdl.handle.net/10803/7468

Schweingruber, F. H. 1990: Anatomie europäischer Hölzer. Ein atlas zur bestimmung europaischer baum, strauch und zwerdstrauchholzer. Verlag Paul Haupt. Bern - Stuttgart.

Servera-Vives, G.; Riera, S.; Picornell-Gelabert, L1.; Moffa-Sánchez, P.; Llergo, Y.; Garcia, A.... y Calvo Trías, M. 2018: "The onset of islandscapes in the Balearic Islands: A study-case of Addaia (northern Minorca, Spain)". Palaeogeography, Palaeoclimatology, Palaeoecology 498: 9-23. https://doi.org/10.1016/j.palaeo.2018.02.015

Waldren, W. H. 1984: "Chalcolithic settlement and baker connections in the Balearic Islands". En W. H. Waldren, R. Chapman, J. Lewthwaite y R. Kennard (eds.): The Deya Conference of Prehistory: early settlement in the Western Mediterranean Islands and their peripheral areas. British Archaeological Reports International Series. Archaeopress. Oxford: 911-965.

Waldren, W. H. 1987: “A Balearic Beaker model. Ferrandell-Oleza, Valldemossa, Mallorca”. En W. H. Waldren y R. C. Kennard (eds.): Bell Beakers of the Western Mediterranean. Definition, interpretation, theory and new site data. British Archaeological Reports International Series. Archaeopress. Oxford: 207-66.

Yll, E. I.; Pantaleón-Cano, J.; Pérez-Obiol, R. y Roure, J. M. 1999: "Cambio climático y transformación del medio durante el Holoceno en las Islas Baleares”. En J. Bernabeu Aubán y T. Orozco Köhler (eds.): Actes del II Congrés del Neolític a la Península Ibèrica (Valencia 1999). Saguntum. Papeles del Laboratorio de Arqueología de Valencia, Extra 2. Valencia: 45-51.

Y11, E. I.; Pérez-Obiol, R. y Julià, R. 1994: "Vegetational change in the Balearic Islands (Spain) during the Holocene". Historical Biology 9: 83-89. https://doi.org/10.1080/10292389409380490

Yll, E. I.; Pérez-Obiol, R.; Pantaleon-Cano, J. y Roure, J. M. 1997: "Palynological Evidence for Climatic Change and Human Activity during the Holocene on Minorca (Balearic Islands)". Quaternary Research 48: 339-347. https://doi.org/10.1006/qres.1997.1925 